\title{
Article \\ A Systems Biology Approach to Identify Essential Epigenetic Regulators for Specific Biological Processes in Plants
}

\author{
Rachel M. McCoy ${ }^{1,2,+} \oplus$, Russell Julian ${ }^{1,2,+}$, Shoban R. V. Kumar ${ }^{1,2}$, Rajeev Ranjan ${ }^{1,2}{ }^{\oplus}$, Kranthi Varala ${ }^{1,2}{ }^{(}$and \\ Ying $\mathrm{Li}^{1,2, *}$ \\ 1 Department of Horticulture and Landscape Architecture, Purdue University, West Lafayette, IN 47907, USA; \\ mccoy26@purdue.edu (R.M.M.); russell.s.julian@gmail.com (R.J.); srajaman@purdue.edu (S.R.V.K.); \\ ranjan9@purdue.edu (R.R.); kvarala@purdue.edu (K.V.) \\ 2 Center for Plant Biology, Purdue University, West Lafayette, IN 47907, USA \\ * Correspondence: li2627@purdue.edu; Tel.: +1-765-494-0104 \\ + These authors contributed to the work equally.
}

Citation: McCoy, R.M.; Julian, R.; Kumar, S.R.V.; Ranjan, R.; Varala, K.; Li, Y. A Systems Biology Approach to Identify Essential Epigenetic Regulators for Specific Biological Processes in Plants. Plants 2021, 10, 364. https://doi.org/10.3390/ plants10020364

Academic Editor: Christel Carles

Received: 5 January 2021

Accepted: 7 February 2021

Published: 13 February 2021

Publisher's Note: MDPI stays neutral with regard to jurisdictional claims in published maps and institutional affiliations.

Copyright: (c) 2021 by the authors. Licensee MDPI, Basel, Switzerland. This article is an open access article distributed under the terms and conditions of the Creative Commons Attribution (CC BY) license (https:// creativecommons.org/licenses/by/ $4.0 /)$.

\begin{abstract}
Upon sensing developmental or environmental cues, epigenetic regulators transform the chromatin landscape of a network of genes to modulate their expression and dictate adequate cellular and organismal responses. Knowledge of the specific biological processes and genomic loci controlled by each epigenetic regulator will greatly advance our understanding of epigenetic regulation in plants. To facilitate hypothesis generation and testing in this domain, we present EpiNet, an extensive gene regulatory network (GRN) featuring epigenetic regulators. EpiNet was enabled by (i) curated knowledge of epigenetic regulators involved in DNA methylation, histone modification, chromatin remodeling, and siRNA pathways; and (ii) a machine-learning network inference approach powered by a wealth of public transcriptome datasets. We applied GENIE3, a machine-learning network inference approach, to mine public Arabidopsis transcriptomes and construct tissue-specific GRNs with both epigenetic regulators and transcription factors as predictors. The resultant GRNs, named EpiNet, can now be intersected with individual transcriptomic studies on biological processes of interest to identify the most influential epigenetic regulators, as well as predicted gene targets of the epigenetic regulators. We demonstrate the validity of this approach using case studies of shoot and root apical meristem development.
\end{abstract}

Keywords: epigenetic regulator; machine learning; gene regulatory network; shoot apical meristem; root apical meristem

\section{Introduction}

In eukaryotes, gene regulation occurs in the context of chromatin and nucleosomes, which is influenced by epigenetic modifications that pack genomic DNA and poise genes for activation or repression [1]. Broadly, epigenetic modifications include DNA methylation (which involves the siRNA silencing pathway), histone modification, and chromatin remodeling. Epigenetic states are dynamically modified by numerous epigenetic regulators that are encoded by specific gene families. For example, histone methylation, a form of histone modification, is added to specific amino acid residues on histone tails through the function of a group of enzymes called histone methyltransferases (HMTs) [2] and removed by the opposing action of another group of enzymes named histone demethylases (HDMs) [3]. Similarly, histone acetylation is maintained by the concerted actions of histone acetyltransferases (HAT) and histone deacetylases (HDAC) [3]. Epigenetic regulators can transform the chromatin landscape of a corresponding suite of genes to modulate their expression and dictate adequate cellular and organismal responses to developmental signals or environmental cues. Indeed, experimental evidence accumulated in the past 20 years established that epigenetic regulators and chromatin modifications play essential 
roles in modulating gene expression in response to developmental and environmental signals [3-8]. For example, SDG8, a H3K36 (lysine 36 of histone subunit 3) methyltransferase, was shown to specifically target hundreds of genes involved in defense, carbon metabolism, and reproductive development to modify their associated histones and gene expression levels [9], and it is required for proper responses to various environmental [10-12] and developmental signals [13]. To date, key epigenetic regulators have been identified for many, but not all, developmental and environmental processes [10,14-19]. Meanwhile, ongoing efforts to detect target genes of epigenetic regulators will continue to expand our knowledge of the specificity of epigenetic regulators $[9,20]$. A knowledge base to link each epigenetic regulator with specific biological processes and genome-wide targets is needed to move toward a genome-wide, systems-level understanding of epigenetic regulation.

Gene regulatory network (GRN) inference is a powerful method to predict regulatory relationships between regulators, genes, and biological processes. GRNs have been used to predict the regulatory relationships between transcription factors (TFs) and their target genes, and the TFs that control the most target genes in a GRN are identified as potential key regulators involved in the corresponding biological process [21,22]. Therefore, just as GRN inference has been used to infer transcriptional regulators [21,22], theoretically, it could be used to identify key epigenetic regulators of a given biological process-a notion which has not been tested, to the best of our knowledge. In eukaryotes, complex interplay between TFs and epigenetic regulators controls the coordinated expression of thousands of genes [23]. However, common approaches for gene network inference often use only TFs as regulators [24-27] and overlook the important layer of epigenetic regulation. In recent years, there has been an exponential accumulation of epigenomics data (e.g., in ENCODE [28]), which has led to emerging efforts to incorporate epigenetic states of genes (e.g., histone methylation status) into network models [29-31]. However, these models have not included the epigenetic regulators that cause these state changes in the resulting network models.

To facilitate the generation of knowledge on the specific function and targets of epigenetic regulators, we developed a bioinformatic pipeline built around GENIE3 [32] to mine large transcriptome datasets and construct tissue-specific GRNs with both epigenetic regulators and transcription factors as predictors or regulators of gene expression. Of the numerous GRN inference tools available [21], the random forest-based machine learning approach of GENIE3 was shown to be the best-performing tool to infer regulatory relationships from large transcriptome datasets [33]. The inferred GRNs can then be intersected with an individual transcriptomic study to identify the most influential epigenetic regulators for the biological processes of interest. Using meristematic development as a case study, we identified key epigenetic regulators that control a network of genes important for developmental processes in meristematic tissues of the shoot apical meristem (SAM) and root apical meristem (RAM), separately. Finally, our network predictions were broadly validated by published experimental data. The work presented here was based on Arabidopsis thaliana transcriptomic data, due to the abundance of this type of data in the public realm, but the described approaches could be applied to other plant systems. This bioinformatics pipeline can be applied to any biological process of interest to greatly speed up hypothesis generation and testing of the roles of epigenetic regulation in controlling plant developmental and environmental responses.

\section{Results}

\subsection{Constructing Gene Regulatory Networks (GRNs) with Epigenetic Regulators}

To probe the influence of epigenetic regulators in controlling GRNs, we first generated a list of known epigenetic regulators in model plant Arabidopsis thaliana. We chose to work with Arabidopsis because of the rich collection of knowledge on epigenetic regulators and a wealth of transcriptomic datasets is available for this model plant species. An extensive literature review was performed, and a list of 286 epigenetic regulators was manually curated based on 186 publications (see Table S1 for the complete list of genes and 
corresponding references). This includes 174 genes encoding epigenetic regulators involved in histone modification, 62 genes in DNA methylation, 30 in chromatin remodeling, and 20 involved in siRNA biogenesis (see Supplemental Results for description of these genes).

Next, we inferred organ-specific gene regulatory networks (GRNs) to determine regulatory relationships between a regulatory gene, i.e., an epigenetic regulator or transcription factor (TF), and its target genes. In detail, 1000 Arabidopsis transcriptomes from shoots and 778 transcriptomes from roots were retrieved from publicly available transcriptome data in SRA (short read archive, NCBI) (Figure 1A). Next, we ran GENIE3 [32], a machine learning algorithm that predicts gene regulatory networks from expression data [32], to infer the GRN for shoots and roots, separately (Figure 1A). Importantly, when running GENIE3, both transcription factors (1717 genes based on AtTFDB [34]) and epigenetic regulators (286 genes curated by this study, Table S1) were used as predictors, whose transcript abundances are used to predict the abundance of other transcripts (Figure 1A). Namely, both TFs and epigenetic regulators are allowed to influence the expression level of genes in the inferred network. The resultant GRNs contain nodes, which are genes including both predictors (TFs or epigenetic regulators) and regulated genes, and edges, where each edge represents a regulatory relationship between a predictor and a regulated gene. Each edge has a weight assigned by GENIE3, as a measurement of the confidence of the prediction [32]. All the edges in resultant shoot and root GRNs were ranked by weight (largest to smallest), separately, and the top $10 \%$ of edges were retrieved to generate higher confidence GRNs, referred to as EpiNet hereafter (Figure 1A). The shoot EpiNet includes 31,514 nodes and 6,286,230 edges, while the root EpiNet includes 33,134 nodes and 6,609,470 edges (Supplemental Data 1 and 2). In summary, by taking advantage of well-annotated epigenetic regulators and a wealth of transcriptome data in Arabidopsis, we have generated, with an unbiased approach, GRNs for shoots and roots. These GRNs were trimmed in an unbiased manner (retaining the top 10\% of edges) to control their false positive rate $[22,35,36]$. Importantly, in these GRNs, in addition to TFs that are commonly used as predictors, epigenetic regulators are also included as predictors and thus contribute toward the regulation of a specific set of downstream target genes in the network.

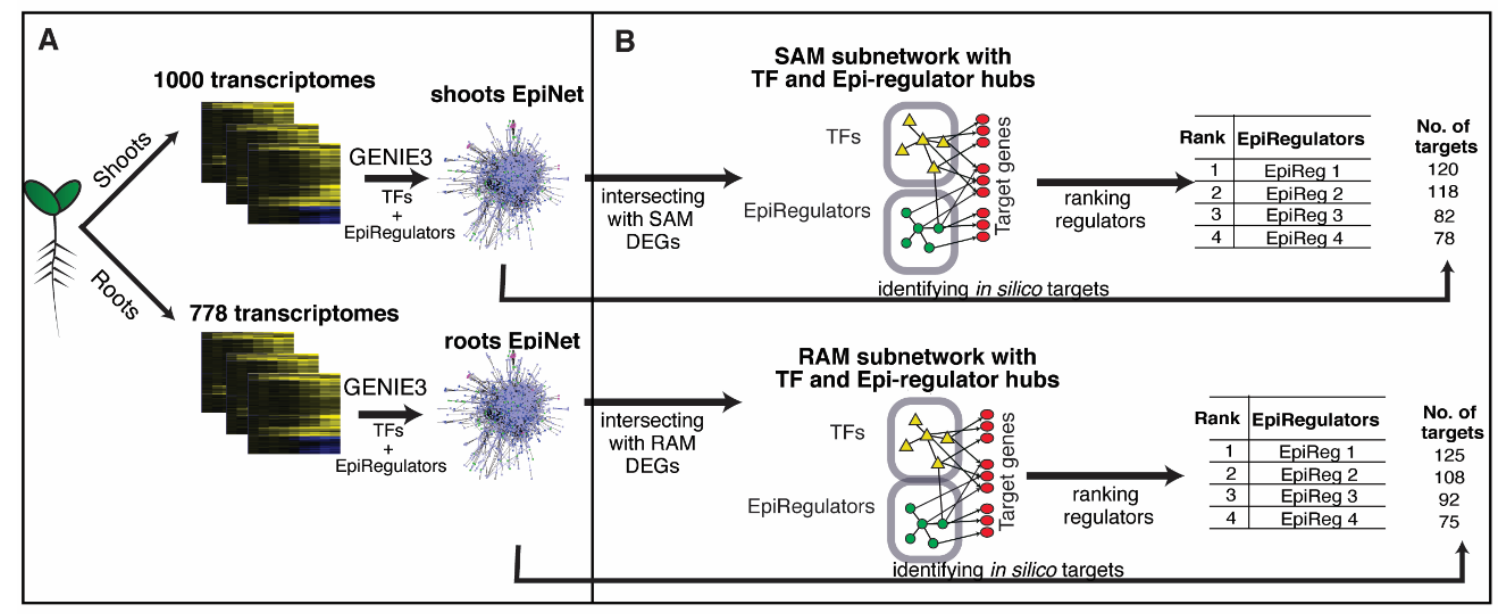

Figure 1. Workflow showing the analysis steps of this study: (A) 1000 transcriptomes from Arabidopsis shoots and 778 transcriptomes from Arabidopsis roots were downloaded from NCBI's SRA (short read archive) database. GENIE3 was applied to construct gene regulatory networks from these transcriptomes for shoots and roots, separately, using both transcription factors (TFs) and epigenetic regulators (EpiRegulators) as predictors of gene expression levels. The resultant gene regulatory networks are named EpiNet, for shoots and roots, separately. (B) EpiNet was intersected with differentially expressed genes (DEGs) identified from individual transcriptomic studies to generate subnetworks relevant to the question of interest-for example, shoot apical meristem (SAM) and root apical meristem (RAM) development. The epigenetic regulators involved in controlling the subnetwork can then be ranked based on their influence on the genes in the subnetwork, to identify the most essential epigenetic regulators for the biological process of interest. For each epigenetic regulator, the in silico predicted targets can be identified in the EpiNet. 


\subsection{Case Study: Identifying Influential Epigenetic Regulators in Meristematic Development}

Next, we tested whether the EpiNet is able to provide informative predictions to understand the role of epigenetic regulators in specific biological processes. As a case study, we focused on the developmental processes of the shoot apical meristem (SAM) and root apical meristem (RAM). Plant meristems are the main area of primary growth and are made up of a collection of undifferentiated pluripotent stem cells, the number and positioning of which are under tight control. The SAM is the source of aboveground post-embryogenesis growth, including reproductive tissues, as it is converted to an inflorescence meristem during floral transition. The stem cells of the SAM are in the center of the meristem in an area termed the central zone (CZ). Surrounding these regions of slowly dividing cells is the peripheral zone (PZ) that gives rise to differentiated tissues. Below the $\mathrm{CZ}$ is the rib meristem (RM) zone that develops into stem tissue. Similarly, the RAM is the source of post-embryogenic root growth. The roots can be divided into the differentiated zone, the elongation zone, and the meristematic zone (which contains the RAM stem cells localized in the quiescent center $(\mathrm{QC})$ ). In general, meristem development has been well studied, and many transcription factors have been reported to perform important functions in maintaining meristematic tissues, including WUSCHEL and KNOTTED1-LIKE HOMEOBOX (KNOX) genes in SAM and WUSCHEL-LIKE HOMEOBOX 5 (WOX5), SCARECROW, SPAT$U L A$, and PLETHORA in RAM (see [37-40] for recent reviews). By contrast, less is known about the roles of epigenetic regulators in SAM and RAM development [41,42]. Overall, SAM and RAM development provide a good model to test the efficacy of our approach.

\subsubsection{Creating Subnetworks Related to SAM and RAM Development Based on Representative Transcriptomic Studies}

The EpiNet was generated without any focus on any particular biological processes and therefore contains transcriptional and epigenetic regulatory information for the whole genome. To generate subnetworks relevant to SAM or RAM development, we first searched for published transcriptomic studies that identified genes involved in SAM or RAM development. We focused on two transcriptomic studies of SAM, Yadav et al. [43] and Tian et al. [44]. In these two studies, cell-layer-specific transcriptomic profiling was performed; therefore, these studies likely have high sensitivity and identified a wide range of genes related to SAM development and function. In Yadav et al. [43], a mutant named apetala1-1; cauliflower1-1 (ap1-1; cal1-1) was used because it produces multiple SAMs [45]. Different cell layers within the SAMs were labeled by expressing fluorescent proteins under the control of promoters known to drive gene expression specifically in those cell layers. For example, the CLV3 promoter was used to label the central zone (CZ), the FILAMENTOUSFLOWER (FIL) promoter was used to label the peripheral zone (PZ), especially the flower organ primordia, and the WUSCHEL (WUS) promoter labeled the rib meristem (RM). Microarray assays were performed to compare the transcriptomes between the three labeled cell layers with fluorescent protein-negative SAM protoplasts as control. This study led to the identification of 2515 differentially expressed genes (DEGs) that are significantly upregulated in at least one of the three cell layers. Similarly, Tian et al. [44] also reported zone-specific transcriptomes from SAM through a different profiling method-translating ribosome affinity purification followed by sequencing (TRAP-Seq). In Tian et al. [44], transgenic plants were created in which the large subunit ribosomal protein L18 was fused with N-terminal His and FLAG tags, driven by zone-specific promoters such as pCLV3, pUFO, and pWUS (for CZ, PZ, and $\mathrm{RM}$ /organizing center (OC), respectively). From the transgenic seedlings, polysomes were immunoprecipitated using antibodies specific for the His or FLAG affinity tags, and the co-precipitated mRNA was then profiled by deep RNA-sequencing. This study identified 2013 genes that are expressed specifically in the CZ, PZ, and RM/OC zones in SAM.

For both studies, the list of identified DEGs was used to query the shoot EpiNet to generate a SAM subnetwork that contains: (i) epigenetic regulators and TFs that are inferred to affect the expression levels of the DEGs identified in Yadav et al. [43] or Tian et al. [44]; (ii) the DEGs that are regulated by the regulators in (i); and (iii) the regulatory relationships 
between (i) and (ii) (Figure 1B). Note that the regulators themselves are not required to be differentially expressed. From Yadav et al. [43], the resulting SAM subnetwork contains 482,082 edges and 4463 nodes, including 1673 TF regulators and 275 epigenetic regulators (Supplemental Data 3). From Tian et al. [44], the generated SAM subnetwork contains 358,047 edges and 3937 nodes, including 1650 TF regulators and 274 epigenetic regulators (Supplemental Data 4).

To generate the RAM subnetwork, we used RAM-specific DEGs reported in Nawy et al. [46]. In Nawy et al. [46], the AGAMOUS-LIKE 42 (AGL42) promoter, which is known to drive quiescent center (QC)-specific expression, was fused to GFP in order to label root cells in the QC. Fluorescent cells from root protoplasts were then sorted, and RNA was extracted and profiled using microarray. The global gene expression profile in the QC cells was compared to that in surrounding tissues, generating a list of 290 QC-specific expressed genes. This list of DEGs was used to query the root EpiNet, generating a RAM subnetwork with 67,533 edges and 2174 nodes, including 1612 TF regulators and 272 epigenetic regulators (Figure 1B; Supplemental Data 5).

2.2.2. The Predicted Top TFs and Epigenetic Regulators Have Reported Roles in Meristem Development

To identify the most influential epigenetic regulators of SAM and RAM subnetworks, the regulators in each subnetwork were further ranked by their weight sum, which represents their net influence on the genes in the subnetwork (see Methods for details). The top ranked regulators with the greatest weight sums are predicted to regulate the largest number of genes in the subnetwork and/or have the highest confidence. We anticipate that some of the most influential regulators of apical meristem development, or indeed any well studied biological process, would have been already identified by traditional genetic approaches. Therefore, we first examined the top ranked regulators, including both TF regulators and epigenetic regulators, to determine whether known regulators of meristematic development are among the top predicted regulators in the SAM or RAM subnetworks. If ranked highly in the predicted list, such known regulators would support the efficacy of our network inference approach.

We first examined the top 25 regulators identified in the two SAM subnetworks. Interestingly, the top 25 regulators identified based on Yadav et al. (Table S2) or Tian et al. (Table S3) share a 64\% overlap (Figure 2A), even though the two lists of DEGs identified from Yadav et al. and Tian et al. only have a much smaller overlap (Figure 2B; an overlap of 362 genes, which represents $14.4 \%$ and $18.0 \%$ of the input DEG lists from Yadav et al. and Tian et al., respectively). Indeed, the regulators of the two SAM subnetworks share high similarity: the ranks (Figure 2C) and the weight sums (Figure 2D) of regulators are highly correlated between the two SAM subnetworks $\left(\mathrm{R}^{2}=0.9212\right.$ for ranks and 0.8875 for weight sums). By contrast, the ranks of regulators are less correlated between the RAM subnetwork and the SAM subnetwork identified based on Yadav et al. (Figure 2E, $\mathrm{R}^{2}=0.1451$ ) or Tian et al. (Figure $2 \mathrm{~F}, \mathrm{R}^{2}=0.1292$ ).

To integrate the ranking of regulators from the analyses of Yadav et al. (Table S2) or Tian et al. (Table S3), a cumulative rank that summarizes the two lists of top 25 regulators was generated using a Rank Sum method (Table S4). In the integrated ranking, the top regulator is HOMOLOGY DEPENDENT GENE SILENCING 1 (HOG1), encoding a S-adenosyl-L-homocysteine hydrolase required for DNA methylation [47]. To the best of our knowledge, a role for HOG1 in meristematic development has not been reported. However, among the remaining top regulators, many regulators have been previously reported to be involved in regulating meristem development. For example, CONSTANS-LIKE 5 (COL5), ranked second, is a member of the CONSTANS transcription factors family [48] and its overexpression was shown to induce flowering [49]. Our analysis thus suggests that COL5 possibly controls the SAM gene network to facilitate the transition of SAM to inflorescence meristem when environmental and/or developmental signals permit. In addition, two members of the KNOTTED-LIKE HOMEOBOX (KNOX) transcription factor family, KNOTTED-LIKE FROM ARABIDOPSIS THALIANA 1 (KNAT1) and KNAT4, are 
ranked fourth and seventh, respectively (Table S4). KNAT1 is primarily expressed in and around the SAM [50], and overexpression of KNAT1 results in lobed leaves with ectopic meristems [51]. KNAT4 is reported to suppress meristematic capacity redundantly with other members of the KNOX family, KNAT3 and KNAT5 [52]. Further, CYCLING DOF FACTOR 3 (CDF3), belonging to the DNA-binding with one finger (DOF)-type transcription factor family, is ranked sixth among the top regulators. CDF3 was shown to repress floral transition [53] and therefore might play a role in regulating the transition of SAM to inflorescent meristem. In addition to identifying previously known TFs involved in meristematic regulation, one epigenetic regulator with reported roles in regulating SAM development was also among the top regulator list (Table S4): SPLAYED (SYD; AT2G28290). SYD is a member of the SWI/SNF2 family of chromatin remodelers and has been shown to be required for meristem development, and syd mutants displayed premature termination of the floral meristem [42].

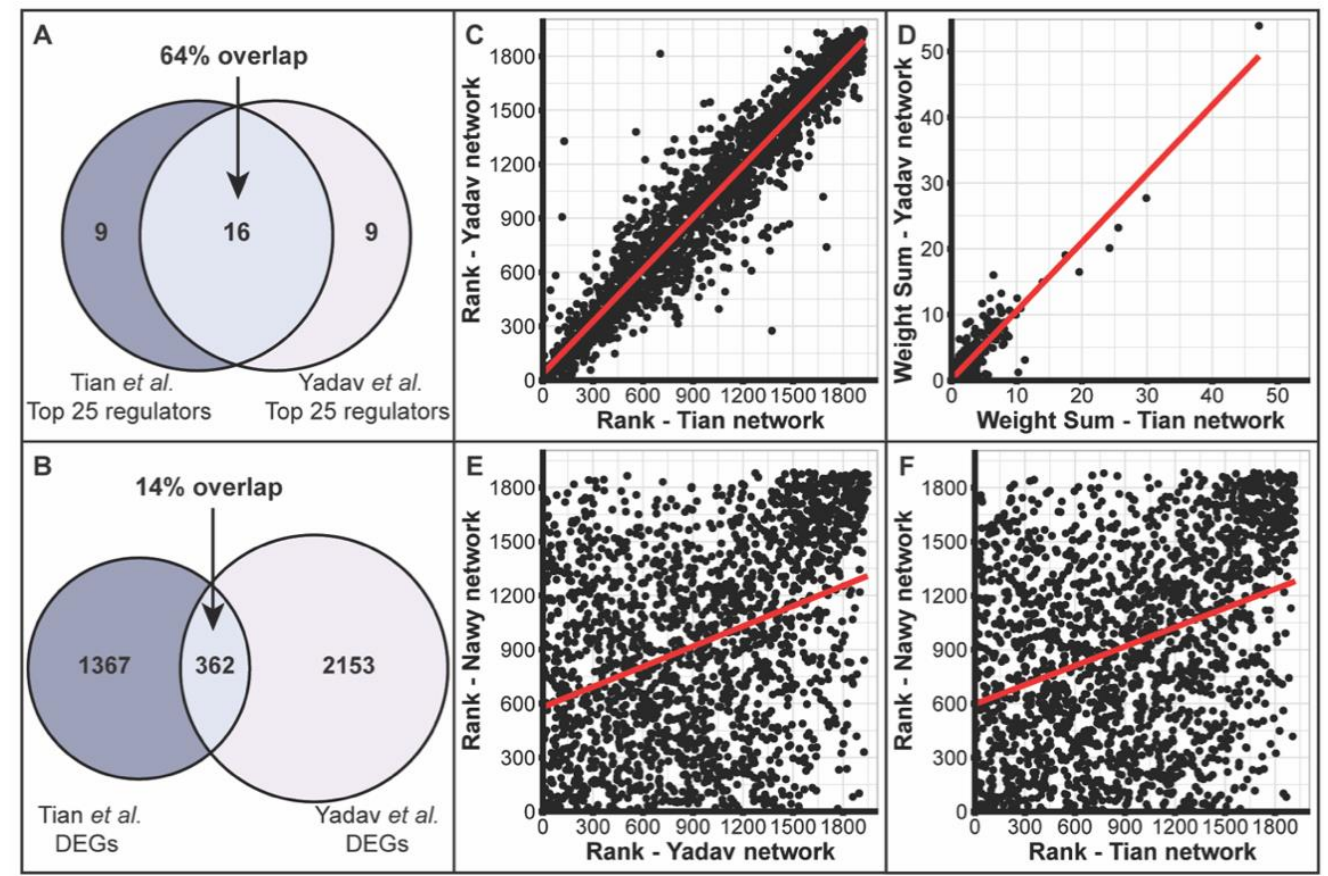

Figure 2. Similar regulators identified for SAM subnetworks generated from Yadav et al. and Tian et al. In total, 16 of the top 25 regulators are shared between the two SAM subnetworks (A), though they share only $<20 \%$ of input DEG genes (B). The rank (C) and weight sum (D) of the two SAM subnetworks are strongly correlated $\left(R^{2}=0.9212\right.$ and 0.8875 , respectively). By contrast, the correlation of regulator ranks between the RAM subnetwork (generated from Nawy et al.) with either the SAM subnetwork generated using Yadav et al. (E) or Tian et al. $(\mathbf{F})$ is much weaker $\left(R^{2}=0.1292\right.$ and 0.1451 , respectively).

For RAM development, similarly, we examined the top ranked regulators, including both TF regulators and epigenetic regulators (Table S5), and identified multiple regulators with known functions in meristematic development. The top regulator in the RAM network is WHIRLY 2 (WHY2), a member of the WHIRLY transcription factor family of single-stranded DNA binding proteins required for maintaining mitochondria. To the best of our knowledge, it is unknown whether WHY2 is involved in regulating RAM development. The second most influential regulator is SPATULA (SPT). SPT encodes a basic-helix-loop-helix family (bHLH) transcription factor first shown to be involved in floral development $[54,55]$. SPT was also reported as a negative regulator of organ size in leaves [56], cotyledons [57], and roots [58]. In roots, specifically, spt mutants have an increased number of cells in the QC and longer roots, suggesting that SPT is a repressor of cell division in RAM [58]. STERILE APETALA (SAP), ranked fourth, encodes an F-box protein that regulates proliferation of cells in the meristemoid, temporary stem cells which give rise to stomata $[59,60]$. The role of SAP in root meristems is therefore interesting and 
invites further investigation. In addition to the above TF regulators, a few epigenetic regulators with known functions in maintaining meristems have been also identified among the list of top 25 regulators (Table S5). BRM (AT2G46020), ranked 13th, encodes a SWI2/SNF2 chromatin remodeler [61] with a known role in RAM. brm mutants have smaller, disordered RAMs as BRM is required for proper maintenance of the QC [61]. Finally, two members of the plant-specific HD2 family of HDACs, HDT1 and HDT4, were among the top 25 regulators. Multiple members of the HD2 HDACs have been shown to regulate cell number in the root meristem [62].

Overall, the top TFs and epigenetic regulators identified in the SAM and RAM subnetworks are enriched with known regulators for meristematic development, including both TF regulators and epigenetic regulators. Our unbiased approach, with no prior knowledge of known regulators, thus assigned high ranks to many of the known regulators. These results provide robust support that our approach is capable of identifying the key regulators of any developmental processes. Therefore, the rest of the highly ranked but uncharacterized regulators are good candidates for novel regulators of SAM/RAM development.

2.2.3. Identifying Highly Influential Epigenetic Regulators and Their Predicted Targets in SAM/RAM GRNs

Since our primary interest is to identify novel epigenetic regulators for specific biological processes, we generated the lists of top 10 epigenetic regulators in the SAM (Tables S6 and S7) and the RAM subnetworks (Table S8) (Figure 1B). For SAM, a cumulative rank that summarizes the two ranked lists based on either Yadav et al. (Table S6) or Tian et al. (Table S7) was generated by Rank Sum (Table S9). These lists provide promising candidates for novel epigenetic regulators of SAM/RAM development.

To further validate the specificity of our network predictions, the in silico networkinferred targets of top epigenetic regulators (referred to as "in silico targets" hereinafter) were retrieved from the EpiNet (Figure 1B; see Methods for details) and compared with experimentally detected targets of these epigenetic regulators, when available. Since the experimental data are often derived from whole plants and not constrained to the SAM and RAM tissues, we compared the experimentally defined targets to the genome-wide targets in EpiNet. The significance of the overlap is estimated using hypergeometric distribution (see Methods). Overall, we observed significant overlap between the in silico targets and experimentally validated targets for many top epigenetic regulators identified in our study (Table 1). For example, SYD is predicted to regulate 6797 genes in the shoot EpiNet. A transcriptomic study performed with the Arabidopsis ATH1 Affymetrix microarray identified 133 genes that are differentially expressed in the syd-2 seedlings compared to wild-type (WT) plants [63]. Remarkably, 40 out of the 133 genes were predicted by our network, representing a significant enrichment of experimentally validated regulatory targets among the in silico targets (enrichment $p$-value $<0.048$ determined by hypergeometric distribution against microarray background) (Table 1). Similarly, two genes encoding ubiquitin-conjugating enzymes (UBCs), $U B C 1$ and $U B C 2$, were found among the top epigenetic regulators of SAM (Table S9). The UBCs are ubiquitin E2 conjugases that physically interact with two RING E3 ligases, HISTONE MONOUBIQUITATION 1 (HUB1) and HUB2, to monoubiquitinate $\mathrm{H} 2 \mathrm{~B}$ histones [64]. UBC1 and UBC2 were shown to regulate flowering time [64], possibly by affecting the transition from SAM to inflorescence meristem. In EpiNet, UBC1 and UBC2 are predicted to regulate 7239 and 6879 genes in the leaves, respectively. The in silico targets of UBC2 overlap significantly with genes that are downregulated in the $u b c 1 / u b c 2$ double mutants compared to WT in the context of salt-stress response [65] (32/82, hypergeometric $p<0.004$, Table 1$)$. Interestingly, the in silico targets of UBC1 do not overlap significantly with the experimentally determined misregulated genes in $u b c 1 / u b c 2(19 / 82$, hypergeometric $p=$ n.s., Table 1$)$, possibly indicating that UBC2 has a more dominant role in salt stress than UBC1. Finally, JUMONJI-C CONTAINING PROTEIN 30 (JMJ30) has 4610 predicted in silico targets in the shoot network, 
which overlap significantly with genes with altered expression in jmj30/jmj32 seedlings compared to WT plants [66] (32/74, hypergeometric $p<0.0005$, Table 1).

Table 1. Validation of in silico target predictions of epigenetic regulators in shoots and roots. For each epigenetic regulator (1st column), the in silico targets from EpiNet (2nd column) were compared with experimentally determined targets (3rd column) to determine the size of overlap (4th column). Hypergeometric testing was used to determine whether the overlap was statistically significant compared to a random background, and the $p$-value is reported (5th column). A brief description of the experimental data and the citation is included in the last column.

\begin{tabular}{|c|c|c|c|c|c|c|}
\hline & $\begin{array}{l}\text { Epigenetic } \\
\text { Regulator }\end{array}$ & $\begin{array}{l}\text { No. of In } \\
\text { Silico } \\
\text { Targets }\end{array}$ & $\begin{array}{c}\text { No. of } \\
\text { Experimental } \\
\text { Targets }\end{array}$ & $\begin{array}{c}\text { No. of } \\
\text { Overlapping } \\
\text { Targets }\end{array}$ & $\begin{array}{l}\text { Overlap } \\
p \text {-Value }\end{array}$ & Sources of Experimental Data \\
\hline \multirow{4}{*}{$\begin{array}{l}\stackrel{\infty}{0} \\
\frac{0}{0}\end{array}$} & SYD & $4936^{1}$ & 133 & 40 & 0.0476 & DEGs in syd-2 seedlings [63] \\
\hline & UBC1 & 7239 & 82 & 19 & 0.7693 & $\begin{array}{l}\text { Salt stress-induced genes with lower expression in } \\
\qquad u b c 1 / u b c 2 \text { [65] }\end{array}$ \\
\hline & UBC2 & 6879 & 82 & 32 & 0.0032 & $\begin{array}{l}\text { Salt stress-induced genes with lower expression in } \\
\qquad u b c 1 / u b c 2 \text { [65] }\end{array}$ \\
\hline & JMJ30 & 4642 & 106 & 32 & 0.0004 & DEGs in jmj30/jmj32 seedlings [66] \\
\hline \multirow{12}{*}{$\begin{array}{l}0 \\
: \\
0\end{array}$} & & & 90 & 38 & 0.0066 & DEGs in meristem zone of $h d t 1 / 2$ root tips [62] \\
\hline & HDT1 & 8145 & 114 & 48 & 0.0027 & DEGs in elongation zone of $h d t 1 / 2$ root tips [62] \\
\hline & & & 42 & 8 & 0.9557 & DEGs in differentiated zone of $h d t 1 / 2$ root tips [62] \\
\hline & & & 90 & 34 & 0.0996 & DEGs in meristem zone of $h d t 1 / 2$ root tips [62] \\
\hline & HDT2 & 8556 & 114 & 42 & 0.1041 & DEGs in elongation zone of $h d t 1 / 2$ root tips [62] \\
\hline & & & 42 & 17 & 0.1220 & DEGs in differentiated zone of $h d t 1 / 2$ root tips [62] \\
\hline & BRM & $4234^{1}$ & 93 & 26 & 0.0436 & Downregulated genes in brm-101 seedlings [63] \\
\hline & \multirow{4}{*}{ BRM } & \multirow{4}{*}{5470} & 4250 & 925 & 0.0003 & DEGs in brm-1 leaves [67] \\
\hline & & & 5278 & 1127 & 0.0008 & ChIP-Seq using $p B R M:: B R M-G F P$ in $b r m-1$ [68] \\
\hline & & & 7761 & 1702 & $<0.0001$ & ChIP-Seq using $p B R M:: B R M-G F P$ in $b r m-1$ [69] \\
\hline & & & 4831 & 1032 & 0.0014 & ChIP-chip study using BRM antibody [70] \\
\hline & PRMT4A & 5130 & 5504 & 1082 & 0.0098 & DEGs in prmt4a/prmt4b seedlings [71] \\
\hline
\end{tabular}

In the roots, similarly, we observed significant overlaps between the in silico targets and experimentally determined targets for top epigenetic regulators. Interestingly, all four members of the plant-specific HD2 family of HDACs (HDT1, HDT2, HDT3, and HDT4) were among the top 10 epigenetic regulators of the RAM subnetwork (Table S8). The regulated genes by HDT1 and HDT2 in roots have been reported in a transcriptomic study comparing $h d t 1,2$ root tips with WT, specifically in a tissue-specific manner for the meristem zone, elongation zone, and differentiated zone separately [62]. Interestingly, the 8145 in silico targets of HDT1 in the root EpiNet significantly overlap with genes differentially expressed in the meristem and elongation zones, but not the differentiated zone, in $h d t 1,2$ root tips (meristem 38/90, hypergeometric $p<0.007$; elongation $47 / 114$, hypergeometric $p<0.003$; differentiated $17 / 42$, hypergeometric $p=$ n.s.; Table 1 ). There was no significant overlap between the experimentally determined regulated genes in $h d t 1,2$ root tips with the 8556 in silico targets of HDT2 (Table 1). Another top epigenetic regulator is the chromatin remodeler BRM, which is predicted to regulate 5470 genes in the root EpiNet. These in silico targets significantly overlap with downregulated genes in seedlings of brm-101 compared to WT [63] (26/93; hypergeometric $p<0.05$ against microarray background, Table 1), as well as DEGs identified in leaves of brm-1 mutants compared to WT [67] (925/4250, hypergeometric $p<8 \times 10^{-4}$, Table 1$)$. More importantly, the in silico targets of $B R M$ also significantly overlap with direct targets of BRM identified in planta by ChIPbased genome-wide approaches in three studies: (i) two ChIP-Seq studies using transgenic lines carrying $\mathrm{p} B R M:: B R M-G F P$ transgene in the brm-1 mutant background (Li et al. [68] 
1127/5278, hypergeometric $p<0.003$, Table 1; Yu et al. [69] 1702/7761, hypergeometric $p<1.7 \times 10^{-7}$, Table 1); and (ii) a ChIP-chip study using BRM antibody [70] (1032/4832, hypergeometric $p<0.004$, Table 1$)$. Finally, PRMT4A is predicted to regulate 5130 genes in the roots and these targets significantly overlap with DEGs identified in the prmt $4 a / p r m t 4 b$ double mutant [71] (1082/5504, hypergeometric $p<0.01$, Table 1).

Overall, we observed significant overlaps between the in silico network-inferred targets and experimentally validated targets, for the top epigenetic regulators identified in our study. These results further support the predictive power of the EpiNet approach. In summary, our study presented an unbiased gene regulatory network with epigenetic regulators incorporated as potential regulators, as well as a bioinformatics pipeline that allows for the identification and prioritization of influential epigenetic regulators for any specific biological process given that a DEG list is available for said biological process.

\section{Discussion}

Epigenetic regulators, working in concert with transcription factors to modify the expression levels of genes, have an emergent role in controlling gene networks underlying developmental and/or environmental responses. However, compared to transcription factors, the regulatory role of epigenetic modifiers is often overlooked in gene network inference. To integrate epigenomic data into networks, recent efforts have incorporated histone modification patterns as attributes of genes in a regulatory network $[29,30]$. Histone modifications have also been used as prior knowledge to improve predictive network models in yeast [31] However, these studies have overlooked the causal influence of epigenetic regulators on the expression levels of their genomic targets. Epigenetic regulators modify the expression level of their genomic targets, which has been shown in published studies in yeast and human $[72,73]$ and in plants $[9,74]$. Specifically, epigenetic regulators could function as an "on" or "off" switch for a gene locus, e.g., via altering chromatin availability or modulating the abundance or quality of transcripts for actively transcribed genes. Epigenetic regulators could be recruited to these target gene loci by interacting with TFs [75] or other cis-motif-binding proteins [76]. Therefore, in our study, epigenetic regulators are allowed the same "predictor" status as TF regulators in gene network modeling. Including epigenetic regulators in gene network models will likely enhance the ability to predict network states under untested conditions.

The input data for our network inference were curated to only include gene expression values from either the shoots or the roots but in each case included a wide range of experimental conditions and stimuli. Therefore, the GRN inference is likely to pick up regulatory interactions that are broadly occurring in that organ and are not limited to a biological process of interest. No prior information was provided on the relative importance of regulators or the strength of known regulations to our approach, thus making it unbiased. As a result, it is able to predict known and unknown regulators of any biological process occurring in that organ. In our case study, the appropriate selection of a well-defined target set of genes, e.g., the genes involved in SAM/RAM maintenance and development, allows for the identification of TF and epigenetic regulators even when the underlying transcriptome data were not specifically chosen from meristematic tissues. Thus, the EpiNet approach was able to predict a GRN reasonably well in an untested, or at least underrepresented, condition of apical meristem maintenance.

In our study, we used DEGs generated from two different studies of SAM development, Yadav et al. [43] and Tian et al. [44], to create two SAM subnetworks. The two lists of DEGs only have a modest overlap, $<20 \%$, possibly reflecting the different experimental conditions (genotypes, biochemical methods for transcriptomic profiling, etc.) and/or informatics pipelines between the two studies (Figure 2B). Surprisingly, from the two reasonably different lists of DEGs, we identified highly similar regulators, evidenced by the $64 \%$ overlap of top 25 regulators (Figure 2A). Moreover, we observed a high correlation of ranks of regulators between the two subnetworks (Figure 2C). This cannot be explained merely by a dominant contribution from the overlapped 362 DEGs on the ranking of the 
regulators (Figure 2B), as in each case, the "unique" DEGs contribute to $85 \%$ and $82 \%$ of the total weight of edges of the subnetworks from Yadav et al. and Tian et al., respectively. Similarly, one might argue that a hub regulator in the whole EpiNet will likely remain a hub in any subnetwork, thus leading to the similar ranking of regulators between the two SAM subnetworks, but this does not appear to be the explanation here because the ranking of regulators in RAM is quite different (Figure 2E,F). Our observation likely suggests that although the identification of DEGs in a particular transcriptomic study could be sensitive to the specific experimental condition and biochemical/informatics protocols applied, the identification of the upstream epigenetic regulators, by contrast, is more robust.

Our analysis has generated insights into the epigenetic regulators underlying the complex developmental processes of the SAM and RAM. For example, all four members of the plant-specific HD2 family of HDACs (HDT1, 2, 3, and 4) were among the top 10 epigenetic regulators in the RAM subnetwork (Table S8). Similarly, in the SAM subnetwork, HDT1 and HDT2 are among the top epigenetic regulators (Table S9). This family of HDACs is unique in that it is plant-specific $[77,78]$, and they are preferentially expressed in inflorescence tissues and relevant to environmental stresses $[62,77,79,80]$. HDT1 and HDT2 have been shown to regulate cell number in the root meristem [62]. Now, our analysis suggests that this family of HDACs is involved in regulating both SAM and RAM, possibly to alter the histone acetylation status of genes involved in meristematic development in response to environmental changes, potentially serving as an underlying mechanism of developmental plasticity to environmental changes in plants.

To validate our network prediction, we compared predicted in silico targets and experimentally detected targets, mostly determined through transcriptomic assays (Table 1). For multiple epigenetic regulators, we observed a significant overlap between the two types of target genes (in silico predicted vs. experimentally detected), suggesting that the computational predictions are comparable to the in planta scenarios. For a limited number of epigenetic regulators (e.g., BRM), their targets have also been identified by ChIP-based approaches. These ChIP-Seq and ChIP-chip studies have not been used in the construction of the EpiNet, which was built based on transcriptome data. Therefore, the significant overlaps between the in silico targets and the experimental targets identified by ChIP provide completely independent proof that the in silico predicted targets are likely enriched with bona fide targets. In addition, the overlaps of genes in some comparisons were likely underestimated, due to the limitation of experimental conditions. For example, transcriptomic profiling in Arabidopsis was often performed with whole seedlings; therefore, the transcriptomes are likely dominated by gene expression profiles from the shoots, with minimal representation from the roots. Therefore, the comparison of DEGs from such studies with the predicted targets in root EpiNet likely contains many false negatives. To reduce the false negative rate, future functional genomics studies should be performed in the roots to determine the specific gene targets of the epigenetic regulator in the roots. Indeed, the difference between the predicted targets and the experimentally validated targets could be caused by multiple reasons: (i) EpiNet predicts a full range of targets across multiple developmental stages and growth conditions, since the GRNs are based on hundreds to thousands of transcriptomic studies performed under various conditions. By contrast, the experimentally validated targets are limited to the specific developmental stages and growth conditions assayed in those particular experiments; (ii) the experimentally determined targets are sometimes limited by the platform used, e.g., microarray; (iii) the number of experimentally determined targets is also affected by the specific statistical cutoff used in the study; and (iv) additionally, GRN inference itself tolerates a modest to high false positive rate in order to improve recall (i.e., number of true positives identified) [22,33]. Nonetheless, our EpiNet approach demonstrates the strength of GRN-based, data-driven approaches in identifying functions of epigenetic regulators.

Overall, we expect that the EpiNet database and pipeline presented in this study could be utilized by the plant community in a couple of ways: (i) the EpiNet can be intersected with DEGs generated through any transcriptomic study relevant to any specific 
biological process of interest, to identify the epigenetic regulators that are most important for this process, in order to speed up hypothesis generation and testing; (ii) since experimental determination of genome-wide targets of epigenetic regulators is time- and resource-consuming, as a preliminary study, any epigenetic regulator of interest could be queried in the EpiNet databases to identify the predicted targets for shoots and roots, separately. We used SAM and RAM development as examples, and the described approaches can be expanded to study other processes. Our EpiNet was built with transcriptome data from Arabidopsis to take advantage of the extensive collection of transcriptomes generated for this model plant, as training GRNs generally benefits from a large input dataset of transcriptomes. For other plant systems with $>1000$ transcriptomes available, the approaches reported in this study could be applied to generate a species-specific EpiNet. Otherwise, the Arabidopsis EpiNet could still be queried through ortholog mapping. Overall, knowledge of how different epigenetic regulators regulate specific developmental or environmental processes will greatly expand our understanding of gene regulation in plants.

\section{Materials and Methods}

\subsection{Construction of Tissue-Specific Gene Regulatory Networks Including Epigenetic Regulators}

The shoot and root RNA-Seq datasets from Arabidopsis thaliana were obtained from NCBI SRA. The root set included 778 individual RNA-Seq runs and 1000 individual RNASeq runs were retained for the shoot. In both cases, the expression level of all genes across all runs was used to infer a gene regulatory network using the $\mathrm{R}$ implementation of the GENIE3 algorithm [32] with the parameters $\mathrm{K}=$ all and $\mathrm{nCores}=24$ and using $\mathrm{R}$ version 3.5.1. The computation was performed on a Linux server with 24 cores (Sky lake CPUs @ $2.60 \mathrm{~Hz}), 384 \mathrm{~GB}$ RAM. Each inference job took multiple ( 7-10) days to complete. The set of regulators included the known 1717 TFs in Arabidopsis (AtTFDB [34]) as well as 286 epigenetic regulators described above. In the generated network, the weight of the edge is a measure of the confidence in that regulatory edge relative to all the inferred edges in the network [32]. From the ranked list of predictor->target weighted edges provided by GENIE3, edges with the top $10 \%$ of weights were retained for further analysis, referred to as EpiNet. The top $10 \%$ of predicted network is an arbitrary threshold chosen to reduce the low confidence and, often, false positive edges common to network prediction.

\subsection{Creating Subnetworks of SAM and RAM Development and Identifying the Top Regulators}

Genes specifically expressed in the RAM (Nawy et al. [46]) or SAM (Yadav et al. [43] and Tian et al. [44]) were used to filter the EpiNet generated by GENIE3. Each edge of EpiNet (i.e., each line of the GENIE3 output) includes a Regulatory Gene (i.e., regulator or predictor), a Target Gene, and a Weight. To generate SAM- or RAM-specific subnetworks, the shoot or root EpiNet was filtered to only include edges where the Target Gene was in the experimentally determined DEG list. Next, an in-house python script was run to sum the weight of all edges in the subnetwork for every regulator to generate a weight sum. Regulatory genes were then ranked by weight sum from highest to lowest. To determine the top 10 predicted epigenetic regulators, the list of epigenetic regulators (Table S1) was used to filter the rank of Regulatory Genes.

\subsection{Assessing the Significance of the Overlap between In Silico Predicted Targets and Experimentally Validated Targets of Influential Epigenetic Regulators}

To determine the in silico targets of an epigenetic regulator, an in-house python script was used to select all edges in the shoot or root EpiNet, where the Regulatory Gene is the epigenetic regulator of interest. The collection of the Target Genes from these selected edges then constitutes the in silico targets of the epigenetic regulator. The significance of overlap between in silico and experimental targets was determined using hypergeometric distribution in R statistical programming software (phyper; lower.tail = FALSE for overrepresentation). Unless otherwise mentioned, the total number of genes in Arabidopsis was used as background. When experimental data were from a microarray experiment, in silico 
targets were filtered to only include genes presented on the microarray, and the number of probes was used as background.

\section{Conclusions}

We present EpiNet, a bioinformatics resource for inferring organ-specific regulatory relationships between epigenetic regulators and their targets. EpiNet could be used to identify important epigenetic regulators of a specific biological process, as well as regulatory targets of these epigenetic regulators, thus facilitating generation of hypotheses on epigenetic regulation. EpiNet utilizes the well-annotated genome and wealth of available transcriptome studies from Arabidopsis, but this approach could be used in other plants with sufficient transcriptome data.

Supplementary Materials: The following are available online at https:/ /www.mdpi.com/2223-774 7/10/2/364/s1: Supplemental results: Description and literature review of epigenetic regulators used as predictors; Supplementary data S1: Shoot EpiNet; Supplementary data S2: Root EpiNet; Supplementary data S3: SAM subnetwork based on DEGs from Yadav et al.; Supplementary data S4: SAM subnetwork based on DEGs from Tian et al.; Supplementary data S5: RAM subnetwork based on DEGs from Nawy et al.; Supplementary Table S1: List of epigenetic regulators used in this study and references; Supplementary Table S2: Top 25 regulators in the SAM subnetwork generated from Yadav et al. 2009; Supplementary Table S3: Top 25 regulators in the SAM subnetwork generated from Tian et al. 2019; Supplementary Table S4: Cumulative ranking of the top regulators from the SAM subnetworks generated from Yadev et al. and Tian et al.; Supplementary Table S5: Top 25 regulators in the RAM subnetwork generated from Nawy et al. 2009; Supplementary Table S6: Top 10 epigenetic regulators in the SAM subnetwork generated from Yadav et al. 2009; Supplementary Table S7: Top 10 epigenetic regulators in the SAM subnetwork generated from Tian et al. 2019; Supplementary Table S8: Top 10 epigenetic regulators in the RAM subnetwork generated from Nawy et al. 2008; Supplementary Table S9: Cumulative ranking of the top epigenetic regulators in the SAM subnetworks generated from Yadav et al. and Tian et al.

Author Contributions: Conceptualization, K.V. and Y.L.; Data curation, R.M.M., R.J. and R.R.; Formal analysis, R.M.M. and S.R.V.K.; Funding acquisition, K.V. and Y.L.; Methodology, R.J., S.R.V.K., K.V. and Y.L.; Project administration, Y.L.; Supervision, K.V. and Y.L.; Validation, R.M.M.; Visualization, R.M.M. and Y.L.; Writing—original draft, R.M.M., R.J., K.V. and Y.L.; Writing—review and editing, R.M.M., R.J., R.R., K.V. and Y.L. All authors have read and agreed to the published version of the manuscript.

Funding: This material is based upon work supported by the U.S. Department of Energy, Office of Science, Office of Biological and Environmental Research (BER), under Award Number DE-SC0020399. This research is also supported by USDA National Institute of Food and Agriculture Hatch project numbers 1013620 to Y.L.

Data Availability Statement: Data supporting reported results can be found in supplemental materials listed above.

Acknowledgments: We thank Somaiah Balekuttira for his technical assistance in data collection and programming.

Conflicts of Interest: The authors declare no conflict of interest.

\section{References}

1. Felsenfeld, G. A Brief History of Epigenetics. Cold Spring Harb. Perspect. Biol. 2014, 6, a018200. [CrossRef]

2. Alvarez-Venegas, R.; Avramova, Z. SET-Domain Proteins of the Su(Var)3-9, E(z) and Trithorax Families. Gene 2002, 285, $25-37$. [CrossRef]

3. Yuan, L.; Liu, X.; Luo, M.; Yang, S.; Wu, K. Involvement of Histone Modifications in Plant Abiotic Stress Responses. J. Integr. Plant Biol. 2013, 55, 892-901. [CrossRef]

4. Brusslan, J.A.; Alvarez-Canterbury, A.M.R.; Nair, N.U.; Rice, J.C.; Hitchler, M.J.; Pellegrini, M. Genome-Wide Evaluation of Histone Methylation Changes Associated with Leaf Senescence in Arabidopsis. PLoS ONE 2012, 7, e33151. [CrossRef]

5. Maze, I.; Covington, H.E.; Dietz, D.M.; LaPlant, Q.; Renthal, W.; Russo, S.J.; Mechanic, M.; Mouzon, E.; Neve, R.L.; Haggarty, S.J.; et al. Essential Role of the Histone Methyltransferase G9a in Cocaine-Induced Plasticity. Science 2010, 327, 213-216. [CrossRef] [PubMed] 
6. Nimura, K.; Ura, K.; Kaneda, Y. Histone Methyltransferases: Regulation of Transcription and Contribution to Human Disease. J. Mol. Med. 2010, 88, 1213-1220. [CrossRef]

7. Van Dijk, K.; Ding, Y.; Malkaram, S.; Riethoven, J.-J.; Liu, R.; Yang, J.; Laczko, P.; Chen, H.; Xia, Y.; Ladunga, I. Dynamic Changes in Genome-Wide Histone H3 Lysine 4 Methylation Patterns in Response to Dehydration Stress in Arabidopsis Thaliana. BMC Plant Biol. 2010, 10, 238. [CrossRef] [PubMed]

8. Trollope, A.F.; Gutièrrez-Mecinas, M.; Mifsud, K.R.; Collins, A.; Saunderson, E.A.; Reul, J.M.H.M. Stress, Epigenetic Control of Gene Expression and Memory Formation. Exp. Neurol. 2012, 233, 3-11. [CrossRef]

9. Li, Y.; Mukherjee, I.; Thum, K.E.; Tanurdzic, M.; Katari, M.S.; Obertello, M.; Edwards, M.B.; McCombie, W.R.; Martienssen, R.A.; Coruzzi, G.M. The Histone Methyltransferase SDG8 Mediates the Epigenetic Modification of Light and Carbon Responsive Genes in Plants. Genome Biol. 2015, 16, 79. [CrossRef]

10. Berr, A.; Ménard, R.; Heitz, T.; Shen, W.-H. Chromatin Modification and Remodelling: A Regulatory Landscape for the Control of Arabidopsis Defence Responses upon Pathogen Attack: Chromatin Regulation of Plant Defence. Cell. Microbiol. 2012, 14, 829-839. [CrossRef]

11. Li, Y.; Brooks, M.; Yeoh-Wang, J.; McCoy, R.M.; Rock, T.M.; Pasquino, A.; Moon, C.I.; Patrick, R.M.; Tanurdzic, M.; Ruffel, S.; et al. SDG8-Mediated Histone Methylation and RNA Processing Function in the Response to Nitrate Signaling. Plant Physiol. 2020, 182, 215-227. [CrossRef] [PubMed]

12. Lee, S.; Fu, F.; Xu, S.; Lee, S.Y.; Yun, D.-J.; Mengiste, T. Global Regulation of Plant Immunity by Histone Lysine Methyl Transferases. Plant Cell 2016, 28, 1640-1661. [CrossRef]

13. Soppe, W.J.; Bentsink, L.; Koornneef, M. The Early-Flowering Mutant Efs Is Involved in the Autonomous Promotion Pathway of Arabidopsis Thaliana. Development 1999, 126, 4763-4770.

14. Van der Woude, L.C.; Perrella, G.; Snoek, B.L.; van Hoogdalem, M.; Novák, O.; van Verk, M.C.; van Kooten, H.N.; Zorn, L.E.; Tonckens, R.; Dongus, J.A.; et al. HISTONE DEACETYLASE 9 Stimulates Auxin-Dependent Thermomorphogenesis in Arabidopsis Thaliana by Mediating H2A.Z Depletion. Proc. Natl. Acad. Sci. USA 2019, 116, 25343-25354. [CrossRef]

15. Zheng, Y.; Ding, Y.; Sun, X.; Xie, S.; Wang, D.; Liu, X.; Su, L.; Wei, W.; Pan, L.; Zhou, D.-X. Histone Deacetylase HDA9 Negatively Regulates Salt and Drought Stress Responsiveness in Arabidopsis. J. Exp. Bot. 2016, 67, 1703-1713. [CrossRef]

16. Parida, A.P.; Sharma, A.; Sharma, A.K. AtMBD4: A Methylated DNA Binding Protein Negatively Regulates a Subset of Phosphate Starvation Genes. J. Biosci. 2019, 44, 14. [CrossRef]

17. Zhu, J.; Jeong, J.C.; Zhu, Y.; Sokolchik, I.; Miyazaki, S.; Zhu, J.-K.; Hasegawa, P.M.; Bohnert, H.J.; Shi, H.; Yun, D.-J.; et al. Involvement of Arabidopsis HOS15 in Histone Deacetylation and Cold Tolerance. Proc. Natl. Acad. Sci. USA 2008, 105, 4945-4950. [CrossRef] [PubMed]

18. Asensi-Fabado, M.-A.; Amtmann, A.; Perrella, G. Plant Responses to Abiotic Stress: The Chromatin Context of Transcriptional Regulation. Biochim. Biophys. Acta BBA Gene Regul. Mech. 2017, 1860, 106-122. [CrossRef]

19. Colville, A.; Alhattab, R.; Hu, M.; Labbé, H.; Xing, T.; Miki, B. Role of HD2 Genes in Seed Germination and Early Seedling Growth in Arabidopsis. Plant Cell Rep. 2011, 30, 1969. [CrossRef]

20. Xiao, J.; Wagner, D. Polycomb Repression in the Regulation of Growth and Development in Arabidopsis. Curr. Opin. Plant Biol. 2015, 23, 15-24. [CrossRef]

21. Haque, S.; Ahmad, J.S.; Clark, N.M.; Williams, C.M.; Sozzani, R. Computational Prediction of Gene Regulatory Networks in Plant Growth and Development. Curr. Opin. Plant Biol. 2019, 47, 96-105. [CrossRef]

22. Varala, K.; Marshall-Colón, A.; Cirrone, J.; Brooks, M.D.; Pasquino, A.V.; Léran, S.; Mittal, S.; Rock, T.M.; Edwards, M.B.; Kim, G.J.; et al. Temporal Transcriptional Logic of Dynamic Regulatory Networks Underlying Nitrogen Signaling and Use in Plants. Proc. Natl. Acad. Sci. USA 2018, 115, 6494-6499. [CrossRef]

23. Karlebach, G.; Shamir, R. Modelling and Analysis of Gene Regulatory Networks. Nat. Rev. Mol. Cell Biol. 2008, 9, 770-780. [CrossRef]

24. Bonneau, R.; Reiss, D.J.; Shannon, P.; Facciotti, M.; Hood, L.; Baliga, N.S.; Thorsson, V. The Inferelator: An Algorithm for Learning Parsimonious Regulatory Networks from Systems-Biology Data Sets de Novo. Genome Biol. 2006, 7, R36. [CrossRef]

25. Krouk, G.; Mirowski, P.; LeCun, Y.; Shasha, D.E.; Coruzzi, G.M. Predictive Network Modeling of the High-Resolution Dynamic Plant Transcriptome in Response to Nitrate. Genome Biol. 2010, 11, R123. [CrossRef] [PubMed]

26. Nachman, I.; Regev, A.; Friedman, N. Inferring Quantitative Models of Regulatory Networks from Expression Data. Bioinformatics 2004, 20, i248-i256. [CrossRef]

27. Yosef, N.; Shalek, A.K.; Gaublomme, J.T.; Jin, H.; Lee, Y.; Awasthi, A.; Wu, C.; Karwacz, K.; Xiao, S.; Jorgolli, M.; et al. Dynamic Regulatory Network Controlling TH17 Cell Differentiation. Nature 2013, 496, 461-468. [CrossRef]

28. Weinstock, G.M. ENCODE: More Genomic Empowerment. Genome Res. 2007, 17, 667-668. [CrossRef] [PubMed]

29. Guan, D.; Shao, J.; Deng, Y.; Wang, P.; Zhao, Z.; Liang, Y.; Wang, J.; Yan, B. CMGRN: A Web Server for Constructing Multilevel Gene Regulatory Networks Using ChIP-Seq and Gene Expression Data. Bioinform. Oxf. Engl. 2014, 30, 1190-1192. [CrossRef]

30. Wang, L.Y.; Wang, P.; Li, M.J.; Qin, J.; Wang, X.; Zhang, M.Q.; Wang, J. EpiRegNet: Constructing Epigenetic Regulatory Network from High Throughput Gene Expression Data for Humans. Epigenetics 2011, 6, 1505-1512. [CrossRef]

31. Zheng, J.; Chaturvedi, I.; Rajapakse, J.C. Integration of Epigenetic Data in Bayesian Network. In Pattern Recognition in Bioinformatics; Lecture Notes in Computer Science; Loog, M., Wessels, L., Reinders, M.J.T., de Ridder, D., Eds.; Springer: Berlin/Heidelberg, Germany, 2011; pp. 87-96. ISBN 978-3-642-24854-2. 
32. Huynh-Thu, V.A.; Irrthum, A.; Wehenkel, L.; Geurts, P. Inferring Regulatory Networks from Expression Data Using Tree-Based Methods. PLoS ONE 2010, 5, e12776. [CrossRef]

33. Marbach, D.; Costello, J.C.; Küffner, R.; Vega, N.; Prill, R.J.; Camacho, D.M.; Allison, K.R.; Kellis, M.; Collins, J.J.; Stolovitzky, G. Wisdom of Crowds for Robust Gene Network Inference. Nat. Methods 2012, 9, 796-804. [CrossRef]

34. Palaniswamy, S.K.; James, S.; Sun, H.; Lamb, R.S.; Davuluri, R.V.; Grotewold, E. AGRIS and AtRegNet. A Platform to Link Cis-Regulatory Elements and Transcription Factors into Regulatory Networks. Plant Physiol. 2006, 140, 818-829. [CrossRef] [PubMed]

35. Huang, J.; Zheng, J.; Yuan, H.; McGinnis, K. Distinct Tissue-Specific Transcriptional Regulation Revealed by Gene Regulatory Networks in Maize. BMC Plant Biol. 2018, 18, 111. [CrossRef] [PubMed]

36. Walley, J.W.; Sartor, R.C.; Shen, Z.; Schmitz, R.J.; Wu, K.J.; Urich, M.A.; Nery, J.R.; Smith, L.G.; Schnable, J.C.; Ecker, J.R.; et al. Integration of Omics Networks in a Developmental Atlas of Maize. Science 2016, 353, 814-818. [CrossRef] [PubMed]

37. Kitagawa, M.; Jackson, D. Control of Meristem Size. Annu. Rev. Plant Biol. 2019, 70, 269-291. [CrossRef]

38. Fouracre, J.P.; Poethig, R.S. Lonely at the Top? Regulation of Shoot Apical Meristem Activity by Intrinsic and Extrinsic Factors. Curr. Opin. Plant Biol. 2020, 58, 17-24. [CrossRef]

39. Wang, B.; Smith, S.M.; Li, J. Genetic Regulation of Shoot Architecture. Annu. Rev. Plant Biol. 2018, 69, 437-468. [CrossRef]

40. Drisch, R.C.; Stahl, Y. Function and Regulation of Transcription Factors Involved in Root Apical Meristem and Stem Cell Maintenance. Front. Plant Sci. 2015, 6, 505. [CrossRef]

41. Wu, M.-F.; Yamaguchi, N.; Xiao, J.; Bargmann, B.; Estelle, M.; Sang, Y.; Wagner, D. Auxin-Regulated Chromatin Switch Directs Acquisition of Flower Primordium Founder Fate. eLife 2015, 4, e09269. [CrossRef]

42. Wagner, D.; Meyerowitz, E.M. SPLAYED, a Novel SWI/SNF ATPase Homolog, Controls Reproductive Development in Arabidopsis. Curr. Biol. 2002, 12, 85-94. [CrossRef]

43. Yadav, R.K.; Tavakkoli, M.; Xie, M.; Girke, T.; Venugopala, R.G. A High-Resolution Gene Expression Map of the Arabidopsis Shoot Meristem Stem Cell Niche. Proc. Natl. Acad. Sci. USA 2009, 106, 4941-4946. [CrossRef]

44. Tian, C.; Wang, Y.; Yu, H.; He, J.; Wang, J.; Shi, B.; Du, Q.; Provart, N.J.; Meyerowitz, E.M.; Jiao, Y. A Gene Expression Map of Shoot Domains Reveals Regulatory Mechanisms. Nat. Commun. 2019, 10, 1-12. [CrossRef]

45. Ferrándiz, C.; Gu, Q.; Martienssen, R.; Yanofsky, M.F. Redundant Regulation of Meristem Identity and Plant Architecture by FRUITFULL, APETALA1 and CAULIFLOWER. Dev. Camb. Engl. 2000, 127, 725-734.

46. Nawy, T.; Lee, J.Y.; Colinas, J.; Wang, J.Y.; Thongrod, S.C.; Malamy, J.E.; Birnbaum, K.; Benfey, P.N. Transcriptional Profile of the Arabidopsis Root Quiescent Center. Plant Cell 2005, 17, 1908-1925. [CrossRef]

47. Rocha, P.S.C.F.; Sheikh, M.; Melchiorre, R.; Fagard, M.; Boutet, S.; Loach, R.; Moffatt, B.; Wagner, C.; Vaucheret, H.; Furner, I. The Arabidopsis HOMOLOGY-DEPENDENT GENE SILENCING1 Gene Codes for an S -Adenosyl-1-Homocysteine Hydrolase Required for DNA Methylation-Dependent Gene Silencing. Plant Cell 2005, 17, 404-417. [CrossRef] [PubMed]

48. Robson, F.; Costa, M.M.R.; Hepworth, S.R.; Vizir, I.; Pineiro, M.; Reeves, P.H.; Putterill, J.; Coupland, G. Functional Importance of Conserved Domains in the Flowering-Time Gene CONSTANS Demonstrated by Analysis of Mutant Alleles and Transgenic Plants: Analysis of CONSTANS. Plant J. 2002, 28, 619-631. [CrossRef] [PubMed]

49. Hassidim, M.; Harir, Y.; Yakir, E.; Kron, I.; Green, R.M. Over-Expression of CONSTANS-LIKE 5 Can Induce Flowering in Short-Day Grown Arabidopsis. Planta 2009, 230, 481-491. [CrossRef] [PubMed]

50. Lincoln, C.; Long, J.; Yamaguchi, J.; Serikawa, K.; Hake, S. A Knotted1-like Homeobox Gene in Arabidopsis Is Expressed in the Vegetative Meristem and Dramatically Alters Leaf Morphology When Overexpressed in Transgenic Plants. Plant Cell 1994, 6, 1859-1876. [CrossRef]

51. Chuck, G.; Lincoln, C.; Hake, S. KNAT1 Induces Lobed Leaves with Ectopic Meristems When Overexpressed in Arabidopsis. Plant Cell 1996, 8, 1277-1289. [CrossRef]

52. Furumizu, C.; Alvarez, J.P.; Sakakibara, K.; Bowman, J.L. Antagonistic Roles for KNOX1 and KNOX2 Genes in Patterning the Land Plant Body Plan Following an Ancient Gene Duplication. PLoS Genet. 2015, 11, e1004980. [CrossRef]

53. Fornara, F.; Panigrahi, K.C.S.; Gissot, L.; Sauerbrunn, N.; Rühl, M.; Jarillo, J.A.; Coupland, G. Arabidopsis DOF Transcription Factors Act Redundantly to Reduce CONSTANS Expression and Are Essential for a Photoperiodic Flowering Response. Dev. Cell 2009, 17, 75-86. [CrossRef]

54. Heisler, M.G.; Atkinson, A.; Bylstra, Y.H.; Walsh, R.; Smyth, D.R. SPATULA, a Gene That Controls Development of Carpel Margin Tissues in Arabidopsis, Encodes a BHLH Protein. Dev. Camb. Engl. 2001, 128, 1089-1098.

55. Alvarez, J.; Smyth, D.R. CRABS CLAW and SPATULA, Two Arabidopsis Genes That Control Carpel Development in Parallel with AGAMOUS. Dev. Camb. Engl. 1999, 126, 2377-2386.

56. Ichihashi, Y.; Horiguchi, G.; Gleissberg, S.; Tsukaya, H. The BHLH Transcription Factor SPATULA Controls Final Leaf Size in Arabidopsis Thaliana. Plant Cell Physiol. 2010, 51, 252-261. [CrossRef] [PubMed]

57. Josse, E.-M.; Gan, Y.; Bou-Torrent, J.; Stewart, K.L.; Gilday, A.D.; Jeffree, C.E.; Vaistij, F.E.; Martínez-García, J.F.; Nagy, F.; Graham, I.A.; et al. A DELLA in Disguise: SPATULA Restrains the Growth of the Developing Arabidopsis Seedling. Plant Cell 2011, 23, 1337-1351. [CrossRef]

58. Makkena, S.; Lamb, R.S. The BHLH Transcription Factor SPATULA Regulates Root Growth by Controlling the Size of the Root Meristem. BMC Plant Biol. 2013, 13, 1. [CrossRef] [PubMed] 
59. Li, N.; Liu, Z.; Wang, Z.; Ru, L.; Gonzalez, N.; Baekelandt, A.; Pauwels, L.; Goossens, A.; Xu, R.; Zhu, Z.; et al. STERILE APETALA Modulates the Stability of a Repressor Protein Complex to Control Organ Size in Arabidopsis Thaliana. PLoS Genet. 2018, 14, e1007218. [CrossRef] [PubMed]

60. Wang, Z.; Li, N.; Jiang, S.; Gonzalez, N.; Huang, X.; Wang, Y.; Inzé, D.; Li, Y. SCFSAP Controls Organ Size by Targeting PPD Proteins for Degradation in Arabidopsis Thaliana. Nat. Commun. 2016, 7, 11192. [CrossRef] [PubMed]

61. Yang, S.; Li, C.; Zhao, L.; Gao, S.; Lu, J.; Zhao, M.; Chen, C.-Y.; Liu, X.; Luo, M.; Cui, Y.; et al. The Arabidopsis SWI2/SNF2 Chromatin Remodeling ATPase BRAHMA Targets Directly to PINs and Is Required for Root Stem Cell Niche Maintenance. Plant Cell 2015, 27, 1670-1680. [CrossRef]

62. Li, H.; Torres-Garcia, J.; Latrasse, D.; Benhamed, M.; Schilderink, S.; Zhou, W.; Kulikova, O.; Hirt, H.; Bisseling, T. Plant-Specific Histone Deacetylases HDT1/2 Regulate GIBBERELLIN 2-OXIDASE2 Expression to Control Arabidopsis Root Meristem Cell Number. Plant Cell 2017, 29, 2183-2196. [CrossRef] [PubMed]

63. Bezhani, S.; Winter, C.; Hershman, S.; Wagner, J.D.; Kennedy, J.F.; Chang, S.K.; Pfluger, J.; Su, Y.; Wagner, D. Unique, Shared, and Redundant Roles for the Arabidopsis SWI/SNF Chromatin Remodeling ATPases Brahma and Splayed. Plant Cell 2007, 19, 403-416. [CrossRef]

64. Cao, Y.; Dai, Y.; Cui, S.; Ma, L. Histone H2B Monoubiquitination in the Chromatin of FLOWERING LOCUS C Regulates Flowering Time in Arabidopsis. Plant Cell 2008, 20, 2586-2602. [CrossRef] [PubMed]

65. Sun, Y.; Zhao, J.; Li, X.; Li, Y. E2 Conjugases UBC1 and UBC2 Regulate MYB42-mediated SOS Pathway in Response to Salt Stress in Arabidopsis. New Phytol. 2020, 227, 455-472. [CrossRef] [PubMed]

66. Abscisic Acid-Dependent Histone Demethylation during Postgermination Growth Arrest in Arabidopsis—Wu—2019—Plant, Cell \& Environment-Wiley Online Library. Available online: https://onlinelibrary.wiley.com/doi/abs/10.1111/pce.13547 (accessed on 27 December 2020).

67. Torres, E.S.; Deal, R.B. The Histone Variant H2A.Z and Chromatin Remodeler BRAHMA Act Coordinately and Antagonistically to Regulate Transcription and Nucleosome Dynamics in Arabidopsis. Plant J. 2019, 99, 144-162. [CrossRef]

68. Li, C.; Gu, L.; Gao, L.; Chen, C.; Wei, C.-Q.; Qiu, Q.; Chien, C.-W.; Wang, S.; Jiang, L.; Ai, L.-F.; et al. Concerted Genomic Targeting of H3K27 Demethylase REF6 and Chromatin-Remodeling ATPase BRM in Arabidopsis. Nat. Genet. 2016, 48, 687-693. [CrossRef]

69. Yu, Y.; Liang, Z.; Song, X.; Fu, W.; Xu, J.; Lei, Y.; Yuan, L.; Ruan, J.; Chen, C.; Fu, W.; et al. BRAHMA-Interacting Proteins BRIP1 and BRIP2 Are Core Subunits of Arabidopsis SWI/SNF Complexes. Nat. Plants 2020, 6, 996-1007. [CrossRef] [PubMed]

70. Archacki, R.; Yatusevich, R.; Buszewicz, D.; Krzyczmonik, K.; Patryn, J.; Iwanicka-Nowicka, R.; Biecek, P.; Wilczynski, B.; Koblowska, M.; Jerzmanowski, A.; et al. Arabidopsis SWI/SNF Chromatin Remodeling Complex Binds Both Promoters and Terminators to Regulate Gene Expression. Nucleic Acids Res. 2017, 45, 3116-3129. [CrossRef]

71. Hernando, C.E.; Sanchez, S.E.; Mancini, E.; Yanovsky, M.J. Genome Wide Comparative Analysis of the Effects of PRMT5 and PRMT4/CARM1 Arginine Methyltransferases on the Arabidopsis Thaliana Transcriptome. BMC Genom. 2015, 16, 192. [CrossRef] [PubMed]

72. Bracken, A.P.; Dietrich, N.; Pasini, D.; Hansen, K.H.; Helin, K. Genome-wide Mapping of Polycomb Target Genes Unravels Their Roles in Cell Fate Transitions. Genes Dev. 2006, 20, 1123-1136. [CrossRef]

73. Robyr, D.; Suka, Y.; Xenarios, I.; Kurdistani, S.K.; Wang, A.; Suka, N.; Grunstein, M. Microarray Deacetylation Maps Determine Genome-Wide Functions for Yeast Histone Deacetylases. Cell 2002, 4. [CrossRef]

74. Merini, W.; Romero-Campero, F.J.; Gomez-Zambrano, A.; Zhou, Y.; Turck, F.; Calonje, M. The Arabidopsis Polycomb Repressive Complex 1 (PRC1) Components AtBMI1A, B, and C Impact Gene Networks throughout All Stages of Plant Development. Plant Physiol. 2017, 173, 627-641. [CrossRef]

75. Weiste, C.; Dröge-Laser, W. The Arabidopsis Transcription Factor BZIP11 Activates Auxin-Mediated Transcription by Recruiting the Histone Acetylation Machinery. Nat. Commun. 2014, 5, 1-12. [CrossRef]

76. Zhou, Y.; Wang, Y.; Krause, K.; Yang, T.; Dongus, J.A.; Zhang, Y.; Turck, F. Telobox Motifs Recruit CLF/SWN-PRC2 for H3K27me3 Deposition via TRB Factors in Arabidopsis. Nat. Genet. 2018, 50, 638-644. [CrossRef]

77. Luo, M.; Wang, Y.-Y.; Liu, X.; Yang, S.; Lu, Q.; Cui, Y.; Wu, K. HD2C Interacts with HDA6 and Is Involved in ABA and Salt Stress Response in Arabidopsis. J. Exp. Bot. 2012, 63, 3297-3306. [CrossRef]

78. Pandey, R. Analysis of Histone Acetyltransferase and Histone Deacetylase Families of Arabidopsis Thaliana Suggests Functional Diversification of Chromatin Modification among Multicellular Eukaryotes. Nucleic Acids Res. 2002, 30, 5036-5055. [CrossRef]

79. Han, Z.; Yu, H.; Zhao, Z.; Hunter, D.; Luo, X.; Duan, J.; Tian, L. AtHD2D Gene Plays a Role in Plant Growth, Development, and Response to Abiotic Stresses in Arabidopsis Thaliana. Front. Plant Sci. 2016, 7, 310. [CrossRef]

80. Hollender, C.; Liu, Z. Histone Deacetylase Genes in Arabidopsis Development. J. Integr. Plant Biol. 2008, 50, 875-885. [CrossRef] 